\title{
Potenciais evocados auditivos do tronco encefálico na doença de Parkinson: revisão sistemática
}

\author{
Brainstem auditory evoked potentials in Parkinson's disease: systematic review
}

\author{
Márcia da Silva Lopes ${ }^{1}$, Maysa Rabelo², Ana Paula Corona ${ }^{3}$, \\ Ana Caline Nóbrega da Costa ${ }^{4}$, Roberto Paulo Correia de Araújo ${ }^{5}$ \\ ${ }^{1}$ Fonoaudióloga. Doutoranda do Programa de Pós-Graduação \\ Processos Interativos dos Órgãos e Sistemas, Universidade Federal da Bahia \\ ${ }^{2}$ Fonoaudióloga Clínica \\ ${ }^{3}$ Professora Adjunto. Departamento de Fonoaudiologia, ICS - UFBA \\ ${ }^{4}$ Professora Adjunto. Departamento de Fonoaudiologia. Programa de Pós-Graduação \\ Processos Interativos dos Órgãos e Sistemas, ICS - UFBA \\ ${ }^{5}$ Professor Titular. Departamento de Biofunção. Programa de Pós-Graduação \\ Processos Interativos dos Órgãos e Sistemas, ICS - UFBA
}

\begin{abstract}
Resumo
Introdução: A doença de Parkinson (DP) é uma enfermidade neurodegenerativa associada ao envelhecimento. Os comprometimentos auditivos, embora frequentes em idosos, não são claramente estabelecidos na doença. Objetivo: Identificar a presença de processos fisiopatológicos na condução neural das vias auditivas aferentes na DP. Metodologia: Trata-se de uma revisão sistemática, conduzida a partir da identificação de estudos nas bases de dados PUBMED, Web of Science, SCOOPUS, MEDLINE, LILACS, SciELO e Cochrane, utilizando a combinação dos termos Parkinson's disease, hearing, auditory perception, hearing disorders, hearing tests, auditory processing e auditory evoked potentials. Foram incluídos artigos em inglês, português ou espanhol que investigaram os Potenciais Evocados Auditivos do Tronco Encefálico (PEATE) em sujeitos com DP. Realizou-se a descrição dos resultados e análise da qualidade metodológica dos estudos. Resultados: Dos 622 artigos identificados, foram selecionados 11 estudos publicados entre 1981 e 2012. Em sete estudos foi verificado prolongamento das latências do PEATE nos sujeitos com DP em relação aos controles. Por outro lado, em quatro investigações as respostas dos sujeitos com DP foram classificadas como normais. Verificou-se fragilidades no delineamento metodológico dos estudos analisados quanto à seleção dos sujeitos, acurácia da exposição, determinação do desfecho principal e controle de variáveis confundidoras. Conclusão: A presença de alterações na condução neuroeletrofisiológica na DP é controversa, havendo necessidade de estudos conduzidos com maior rigor metodológico quanto ao desfecho de análise, seleção dos sujeitos e controle de variáveis confundidoras. O presente estudo contribui com informações que podem favorecer decisões metodológicas para o desenvolvimento de investigações futuras sobre o tema.

Palavras-chave: Doença de Parkinson. Potenciais Evocados Auditivos do Tronco Encefálico. Transtornos da audição. Vias auditivas. Envelhecimento.

Abstract

Background: Parkinson's disease (PD) is a neurodegenerative disorder associated with aging. The hearing disorders, although frequent in the elderly, are not clearly established by the disease. Objective: Identifying the presence of pathophysiological processes in neural conduction of afferent auditory pathways in PD. Methodology: This is a systematic review, conducted from the identification of studies in the databases PubMed, Web of Science, SCOOPUS, MEDLINE, LILACS, SCiELO and Cochrane, using a combination of the terms "Parkinson's disease", "hearing", "auditory perception", "hearing disorders", "hearing tests", "auditory processing" and "auditory evoked potentials." Articles were included in English, Portuguese or Spanish investigating the Brainstem Auditory Evoked Potentials (BAEP) in individuals with PD. Description and analysis of the results of the methodological quality of the studies was carried out. Results: Of 622 identified articles, 11 studies were selected, published between 1981 and 2012. In seven studies was found prolonged latency of BAEP in individuals with PD compared to controls. Moreover, four investigations into responses of individuals with PD were classified as normal. There are weaknesses in the methodological design of the studies analyzed for the selection of individuals, accuracy of exposure, determining the primary outcome and control of confounding variables. Conclusion: The presence of changes in neuroelectrophysiological conduction into PD is controversial, requiring larger studies conducted with methodological rigor regarding the outcome of analysis, selection of individuals and control of confounding variables. This study provides information that may favor methodological decisions for the development of future research on the topic.
\end{abstract}

Keywords: Parkinson Disease. Evoked Potentials, Auditory, Brain Stem. Hearing disorders. Auditory pathways. Aging.

Correspondência / Correspondence: Márcia da Silva Lopes. Instituto de Ciências da Saúde, Universidade Federal da Bahia. Avenida Reitor Miguel Calmon, S/N. Vale do Canela. Sala 400. Salvador-BA, Brasil. Telefone: +55713283.8959 


\section{INTRODUÇÃO}

O envelhecimento da população mundial é uma realidade crescente e preocupante na área da saúde. No Brasil, dados do Censo do IBGE ${ }^{1}$ apontam que o número de brasileiros na faixa etária com mais de 65 anos avançou de 5,9\% em 2000 para 7,4\% em 2010. Sabe-se que o envelhecimento está associado a doenças neurodegenerativas, como a doença de Parkinson (DP), que é uma enfermidade crônica e acomete $1,8 \%$ de indivíduos acima de 65 anos, chegando a $2,6 \%$ em populações idosas acima de 85 $\operatorname{anos}^{2}$.

Historicamente, o comprometimento motor na DP é o aspecto mais claramente descrito e estudado, caracterizado por manifestações clínicas clássicas como a bradicinesia, tremor e rigidez, decorrentes da depleção dos neurônios dopaminérgicos da substância negra dos núcleos da base no tronco encefálico ${ }^{3}$. Os processos neuropatológicos da doença não se restrigem apenas ao sistema neuronal dopaminérgico, sendo identificada uma variedade de outros sintomas resultantes dos processos fisiopatológicos da doença ou do efeito da terapia dopaminérgica, entre os quais se observam comprometimentos autonômicos, neuropsiquiátricos, do sono e sensoriais. Dentre os prejuízos sensoriais, as alterações olfativas, gustativas, dor e distúrbios do ciclo sono-vigília são descritas como parte dos comprometimentos encontrados nesta doença ${ }^{4}$.

Embora sejam frequentes em idosos, os comprometimentos auditivos ainda não foram claramente estabelecidos na DP. Estudos conduzidos no Brasil $^{5}$ e no exterior ${ }^{6}$ apontam para uma prevalência da perda auditiva em torno de $30 \%$ nos sujeitos com idade acima de 70 anos. Sabe-se que esses comprometimentos refletem negativamente nas habilidades comunicativas dos sujeitos, com impacto na interação social e na qualidade de vida, e podem acometer desde estruturas auditivas sensoriais periféricas até vias neuroauditivas periféricas e centrais ${ }^{7,8}$.

Para a avaliação da condução neuroeletrofisiológica das vias auditivas periféricas, o Potencial Evocado Auditivo do Tronco Encefálico (PEATE) se configura como uma importante ferramenta, permitindo identificar a presença de processos fisiopatológicos que comprometam a atividade neural das vias auditivas aferentes, compreendidas entre o nervo vestíbulo-coclear e o tronco encefálico ${ }^{\text {. }}$

Estudos foram conduzidos com o objetivo de investigar os achados eletrofisiológicos com PEATE em sujeitos com DP, contribuindo com informações relevantes para a caracterização de um possível comprometimento auditivo na doença. Desta forma, esta revisão objetiva identificar a presença de processos fisiopatológicos na condução neural das vias auditivas aferentes, compreendidas entre o nervo vestíbulo-coclear e o tronco encefálico, na DP.

\section{MATERIAIS E MÉTODOS}

Trata-se de uma revisão sistemática sobre achados eletrofisiológicos da avaliação da condução nervosa realizada com o PEATE em sujeitos com DP. O objeto de estudo foram publicações em periódicos indexados nas bases de dados eletrônicos PUBMED, Web of Science e SCOOPUS, bem como na MEDLINE, LILACS, SciELO e Cochrane pela Biblioteca Virtual em Saúde- BVS (http://regional. bvsalud.org/php/index.php). A combinação dos termos Parkinson's disease, hearing, auditory perception, hearing disorders, hearing tests, auditory processing e auditory evoked potentials, nos títulos e resumos, foi utilizada para a identificação dos artigos publicados em inglês, português e espanhol.

A busca e seleção dos artigos foram realizadas por dois avaliadores independentes, a partir da leitura dos resumos e, posteriormente, pela leitura dos artigos na íntegra. A divergência quanto à inclusão de artigo no estudo foi estabelecida por um terceiro avaliador independente. Foram incluídos apenas artigos originais publicados no período de 1963 a junho 2013, que investigaram os potenciais evocados auditivos do tronco encefálico em sujeitos com DP. Todos os artigos de revisão de literatura e relatos de caso foram excluídos da análise.

Para cada artigo selecionado foi descrito o delineamento do estudo (local, período, desenho de estudo, características da população e resultados) e realizada a análise da qualidade metodológica das investigações de acordo com os critérios propostos por Downs e Black ${ }^{10}$. Neste protocolo, os autores propõem 27 critérios que abordam a qualidade da informação reportada, validade interna e externa e poder do estudo. Para o presente estudo, foram excluídos 12 destes critérios, pois não são aplicáveis aos tipos de estudos desenvolvidos e selecionados para esta revisão. Desta forma, os critérios considerados foram: 1) Clareza na descrição de hipóteses ou objetivos; 2) Descrição das principais medidas de desfecho na introdução ou método; 3) Descrição clara das características dos pacientes incluídos no estudo; 4) Descrição da distribuição dos principais confundidores entre os grupos; 5) Descrição clara dos principais achados do estudo; 6) Informação sobre estimativas da variabilidade aleatória dos principais desfechos; 7) Informação sobre valores de probabilidade para os principais desfechos; 8) Representatividade dos sujeitos convidados a participar do estudo em relação à população de origem; 9) Representatividade dos sujeitos incluídos no estudo; 10) Indicação de análises que não foram inicialmente planejadas; 11$)$ Testes estatísticos apropriados para medir os principais desfechos; 12) Nível de acurácia das 
medidas utilizadas para os principais desfechos; 13) Seleção de sujeitos nos grupos casos e controles pertencentes à mesma população; 14) Seleção de sujeitos nos diferentes grupos realizada no mesmo período; 15) Estudo com poder suficiente para detectar um efeito importante, com um nível de significância de $5 \%$. O somatório dos pontos atribuídos aos 15 itens pode atingir, no máximo, 20 pontos.

\section{RESULTADOS}

As estratégias de busca nas bases de dados identificaram 1.254 publicações (Figura 1). Destas, $50,4 \%$ representavam artigos presentes concomitantemente nas diferentes bases consultadas. Assim, o título e resumo de 622 publicações foram analisados e selecionados 15 artigos para o presente estudo. Não houve necessidade de um terceiro avaliador para seleção dos artigos.

A maioria das publicações não incluídas $(97,6 \%)$ desenvolveu investigação com outros métodos de avaliação auditiva, abordava aspectos da fala e linguagem de sujeitos com DP ou a aplicabilidade de pistas auditivas para melhor desempenho motor nos parkinsonianos. Dos 15 artigos selecionados, um foi excluí-

Figura 1. Diagrama de fluxo da inclusão dos artigos.

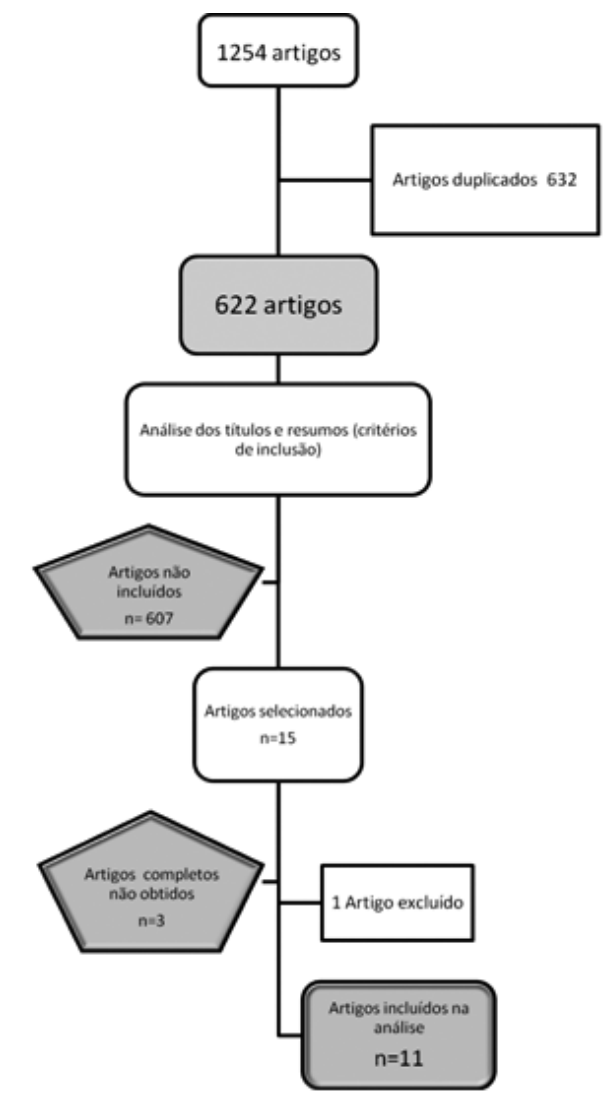

Fonte: Elaboração da autora. do por se tratar de relato de casos e três não foram localizados, na íntegra, no país. Não foram identificados novos artigos a partir das referências das publicações selecionadas. Desta forma, o presente estudo foi conduzido com 11 artigos (Tabela 1).

Dentre os países que contribuíram com as publicações analisadas nesta revisão, dois estudos foram desenvolvidos na Inglaterra, assim como no Japão e Israel. Já no Brasil, Turquia, China, Itália e Estados Unidos foi localizada, respectivamente, uma publicação em cada país.

O estudo mais antigo localizado foi publicado em $1981,{ }^{11}$ havendo mais seis publicações na década

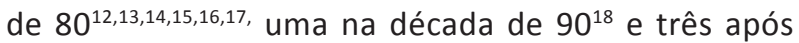
2002. ${ }^{19,20,21}$ Quanto ao desenho de estudo, em nenhuma das publicações analisadas houve a indicação do tipo de estudo realizado e as informações contidas no item métodos não permitiram a classificação do mesmo.

Observa-se que em apenas cinco estudos (41\%) houve a descrição de como o diagnóstico da DP foi estabelecido, sendo considerados aspectos clínicos como a presença de pelo menos dois entre as quatro manifestações motoras da doença ${ }^{16,17,18,19,21}$ e resposta a terapia de reposição com levodopa ${ }^{16,17,21}$.

Entre os critérios de exclusão para a seleção dos sujeitos, a maioria dos estudos consideraram o diagnóstico de outras doenças neurológi$\operatorname{cas}^{11,13,14,15,16,17,18,20,21}$, doença cardiovascular ${ }^{11,13,20,21} \mathrm{e}$ doenças do metabolismo ${ }^{13,14,16,20,21}$.

Adicionalmente, a maior parte dos estudos não informou a população de origem dos sujeitos analisados em relação ao grupo DP e grupo controle $12,14,15,16,17,18,20$. Entre aqueles que indicaram a origem dos sujeitos do grupo DP, foram referenciados serviços de neurologia ${ }^{11,19,21}$ e uma unidade hospita$\operatorname{lar}^{13}$. Dez estudos realizaram avaliação de sujeitos sem a doença para estabelecimento de grupo de comparação, aqui denominado grupo controle, e para tanto, recrutaram cônjuges ${ }^{11,21}$, acompanhantes sem relação genética com os pacientes ${ }^{21}$, pacientes de outras unidades de atendimento ${ }^{13}$ e funcionários do estabelecimento de saúde ${ }^{11,13}$.

O quadro motor nos sujeitos com DP foi caracterizado em seis estudos de acordo com o H\&Y15,17,18,20,21 e a UPDRS ${ }^{21}$. A maioria dos estudos apresentou a média de idade dos sujeitos dos grupos DP e controle, com exceção dos estudos de Gawel et al. ${ }^{11}$, que descrevem a média de idade apenas do grupo de origem dos sujeitos com DP e Vitale et al. ${ }^{21}$ que não informam o dado em relação ao grupo controle.

A análise dos resultados do PEATE foi realizada em grupos constituídos, em média, por 30,25 sujeitos no grupo DP (mínimo 5 e máximo 106) e 29,6 no grupo controle (mínimo 11 e máximo 106). 
Tabela 1. Características dos estudos que analisaram os Potenciais Evocados Auditivos do Tronco Encefálico em sujeitos com doença de Parkinson.

\begin{tabular}{|c|c|c|c|c|c|c|c|}
\hline Referência & Local & $\begin{array}{l}\text { Grupo DP (origem/ } \\
\text { características) }\end{array}$ & $\begin{array}{l}(\mathrm{N}) \\
\mathrm{DP}\end{array}$ & $\begin{array}{l}\text { Controles (origem/ } \\
\text { características) }\end{array}$ & $\begin{array}{c}\text { (N) } \\
\text { Controles }\end{array}$ & Critérios de exclusão & Comentários \\
\hline $\begin{array}{l}\text { Gawel et al, } \\
1981\end{array}$ & Inglaterra & $\begin{array}{l}\text { Sujeitos atendidos } \\
\text { em serviço de } \\
\text { neurologia na Clínica } \\
\text { de doença de } \\
\text { Parkinson }\end{array}$ & 21 & $\begin{array}{l}\text { Cônjuges e funcionários } \\
\text { do hospital com média } \\
\text { de idade } 63 \text { anos; } 11 \\
\text { homens. }\end{array}$ & 12 & $\begin{array}{l}\text { Esclerose múltipla e doença } \\
\text { cardiovascular }\end{array}$ & $\begin{array}{l}\text { Não apresenta os critérios para } \\
\text { diagnóstico da DP. }\end{array}$ \\
\hline $\begin{array}{l}\text { Prasher e } \\
\text { Bannister, } 1986\end{array}$ & Inglaterra & $\begin{array}{l}\text { Média de idade } 58 \\
\text { anos; } 13 \text { homens; }\end{array}$ & 20 & Média de idade 44 anos & 32 & Não informa & $\begin{array}{l}\text { Não apresenta os critérios para } \\
\text { diagnóstico da DP; Todos os sujeitos } \\
\text { avaliados estavam no período on da } \\
\text { medicação. }\end{array}$ \\
\hline $\begin{array}{l}\text { O'Donnel et al., } \\
1987\end{array}$ & $\begin{array}{l}\text { Estados } \\
\text { Unidos }\end{array}$ & $\begin{array}{l}\text { Sujeitos atendidos } \\
\text { em uma unidade } \\
\text { médica ;média de } \\
\text { idade } 65,8 \text { anos }\end{array}$ & 14 & $\begin{array}{l}\text { Funcionários e pacientes } \\
\text { do centro médico; média } \\
\text { de idade } 64,9 \text { anos }\end{array}$ & 11 & $\begin{array}{l}\text { Outras doenças neurológicas, } \\
\text { alterações cárdio-cérebro-vasculares, } \\
\text { alcoolismo ou diabetes } \\
\text { insulinodependentes }\end{array}$ & $\begin{array}{l}\text { Não apresenta os critérios para } \\
\text { diagnóstico da DP; O quadro cognitivo } \\
\text { não foi considerado para a seleção dos } \\
\text { sujeitos; Informa que os indivíduos } \\
\text { estavam em tratamento, porém não } \\
\text { relata se durante as testagens estavam } \\
\text { no período on da medicação. }\end{array}$ \\
\hline $\begin{array}{l}\text { Podoshin et al., } \\
1987\end{array}$ & Israel & $\begin{array}{l}\text { Média de idade } 65,6 \\
\text { anos; } 15 \text { homens }\end{array}$ & 24 & $\begin{array}{l}\text { Média de idade } 56,6 \\
\text { anos }\end{array}$ & 20 & $\begin{array}{l}\text { Hiperlipidemia, diabetes e outras } \\
\text { doenças neurológicas do Tronco } \\
\text { encefálico; Avaliação audiológica } \\
\text { alterada de acordo com a idade }\end{array}$ & $\begin{array}{l}\text { Não apresenta os critérios para } \\
\text { diagnóstico da DP; Analisou os } \\
\text { resultados de } 18 \text { sujeitos em tratamento } \\
\text { com levodopa e } 17 \text { sem tratamento }\end{array}$ \\
\hline $\begin{array}{l}\text { Tachibana, } \\
\text { Takeda e } \\
\text { Sugita, } 1988\end{array}$ & Japão & $\begin{array}{l}\text { H\&Y entre I e V; } \\
\text { Média de idade } 63,3 \\
\text { anos }\end{array}$ & 30 & $\begin{array}{l}\text { Média de idade } 66,7 \\
\text { anos; }\end{array}$ & 22 & $\begin{array}{l}\text { Parkinsonismo secundário e outras } \\
\text { doenças neurológicas }\end{array}$ & $\begin{array}{l}\text { Estabelece claramente os critérios para } \\
\text { diagnóstico da DP idiopática. }\end{array}$ \\
\hline $\begin{array}{l}\text { Fradis et al., } \\
1988\end{array}$ & Israel & $\begin{array}{l}\text { Média de idade } 65,6 \\
\text { anos; } 15 \text { homens; }\end{array}$ & 25 & $\begin{array}{l}\text { Sujeitos com perda } \\
\text { auditiva leve pareada } \\
\text { com os DP; média de } \\
\text { idade } 56,6 \text { anos; }\end{array}$ & 30 & $\begin{array}{l}\text { Hiperlipidemia, diabetes, esclerose } \\
\text { múltipla e outras doenças } \\
\text { neurológicas do Tronco encefálico; } \\
\text { Avaliação audiológica alterada de } \\
\text { acordo com a idade }\end{array}$ & $\begin{array}{l}\text { Não apresenta os critérios para } \\
\text { diagnóstico da DP; Analisou os } \\
\text { resultados de } 18 \text { sujeitos em tratamento } \\
\text { com levodopa e } 17 \text { sem tratamento sem } \\
\text { diferenças entre os grupos quanto a } \\
\text { distribuição da idade }\end{array}$ \\
\hline $\begin{array}{l}\text { Tachibana, } \\
\text { Takeda e } \\
\text { Sugita, } 1989\end{array}$ & Japão & $\begin{array}{l}\text { H\&Y entre le V; } \\
\text { média de idade } 67,3 \\
\text { anos; }\end{array}$ & 44 & Média de idade 69,3 ano & 23 & $\begin{array}{l}\text { Outras doenças neurológicas, } \\
\text { parkinsonismo secundário e abuso de } \\
\text { drogas; }\end{array}$ & $\begin{array}{l}\text { Estabelece claramente os critérios pa ra } \\
\text { diagnóstico da DP idiopática; Analisou o } \\
\text { resultados de } 12 \text { sujeitos com demência } \\
\text { e } 32 \text { sem demência }\end{array}$ \\
\hline $\begin{array}{l}\text { Chia et al., } \\
1995\end{array}$ & China & $\begin{array}{l}\text { Sujeitos atendidos } \\
\text { entre março de } 1992 \\
\text { a agosto de } 1993 ; \\
\text { H\&Y entre I e IV; } \\
\text { média de } 67,2 \text { anos; } \\
19 \text { homens }\end{array}$ & 22 & $\begin{array}{l}\text { Média de idade } 62,6 \\
\text { anos; } 12 \text { homens; }\end{array}$ & 16 & $\begin{array}{l}\text { Parkinsonismo secundário, outras } \\
\text { doenças neurológicas e neuropatia } \\
\text { periférica auditiva ou não, em uso de } \\
\text { antidepressivos, barbitúricos ou } \\
\text { salicilatos. }\end{array}$ & $\begin{array}{l}\text { Critérios para diagnóstico condizentes } \\
\text { com DP idiopática; Analisou os } \\
\text { resultados de } 22 \text { sujeitos em tratamento } \\
\text { com levodopa e } 22 \text { sem tratamento }\end{array}$ \\
\hline $\begin{array}{l}\text { Pineroli et al., } \\
2002\end{array}$ & Brasil & $\begin{array}{l}\text { Sujeitos de um } \\
\text { ambulatório de } \\
\text { neurologia;média de } \\
\text { idade } 64 \text { anos; } 23 \\
\text { homens }\end{array}$ & 32 & Não investiga & $\begin{array}{l}\text { Não } \\
\text { aplica }\end{array}$ & Demência & $\begin{array}{l}\text { Estabelece claramente os critérios para } \\
\text { diagnóstico da DP idiopática. }\end{array}$ \\
\hline $\begin{array}{l}\text { Yýlmaz, et al., } \\
2009\end{array}$ & Turquia & $\begin{array}{l}\text { H\&Y II; média de } \\
\text { idade } 69,9 \text { anos; }\end{array}$ & 20 & $\begin{array}{l}\text { Média de idade } 63,8 ; \\
\text { sem diferenças } \\
\text { estatísticas em relação à } \\
\text { idade }\end{array}$ & 24 & $\begin{array}{l}\text { Diabetes, vasculite, polineuropatia, } \\
\text { esclerose múltipla, } H \& Y \text { acima de } 2,5 \text { e } \\
\text { problemas de equilíbrio. }\end{array}$ & $\begin{array}{l}\text { Não apresenta os critérios para } \\
\text { diagnóstico da DP }\end{array}$ \\
\hline
\end{tabular}

Fonte: elaboração da autora.

Nas tabelas 2 e 3 são apresentados os estudos que identificaram as respostas eletrofisiológicas com o PEATE como normais e alteradas em sujeitos com DP. Em dois estu$\operatorname{dos}^{15,18}$ o protocolo de captação das ondas do PEATE não foi informado. Entre os que apresentaram as informações sobre o protocolo de aquisição, todos utilizaram como estímulo o click, apresentado ipsilateralmente, com velocidade de 10 clicks/ segundo ${ }^{11,12,114,16,17,20,21}$ ou 20 clicks/segundo ${ }^{13,19}$. A intensidade de apresentação do estímulo variou significativamente entre os estudos.

Os parâmetros do PEATE no grupo DP foram considerados dentro da normalidade em quatro estudos (Tabela 2).
Em dois deles, a análise considerou o padrão classificatório dos valores de cada indivíduo em comparação a distribuições normativas estabelecidas pelos achados no grupo controle ${ }^{12} \mathrm{e}$ dados da literatura ${ }^{19}$. O'Donnel et al. ${ }^{13}$ não verificaram diferença entre os grupos DP e controle em relação a latência absoluta da onda I e Vitale et al. ${ }^{21}$ não descrevem o critério de análise dos resultados do PEATE, reportando apenas que não foram observadas alterações no grupo DP.

Por outro lado, foi verificado em sete estudos que as latências absolutas da onda $\mathrm{V}^{12}$ ou as latências interpicos III- $\mathrm{V}^{14,15,16}$ e I-V $\mathrm{V}^{14,15,16,17,18,20}$ estão prolongadas no grupo DP, em comparação ao grupo controle, com medidas estatisticamen- 
Márcia da Silva Lopes et al.

Tabela 2. Estudos que identificaram respostas normais no Potencial Evocado Auditivo do Tronco Encefálico em sujeitos com DP.

\begin{tabular}{|c|c|c|c|c|c|c|c|}
\hline Referência & Protocolo de aquisição PEATE & $\begin{array}{l}\text { Variáveis } \\
\text { analisadas }\end{array}$ & $\begin{array}{c}\text { Critério de } \\
\text { classificação } \\
\text { Normal/Alterado }\end{array}$ & $\begin{array}{l}\text { Comparação entre } \\
\text { grupos }\end{array}$ & Resultados & Pontuação** & Comentários \\
\hline $\begin{array}{l}\text { Prasher e } \\
\text { Bannister, } \\
1986\end{array}$ & $\begin{array}{l}\text { Estímulo ipsilateral, contralateral e } \\
\text { binaural em } 90 \mathrm{dBNA} \text {; velocidade } \\
10 \text { clicks/s }\end{array}$ & $\begin{array}{l}\text { Latências } \\
\text { absolutas das } \\
\text { ondas I, III e V; } \\
\text { razão de } \\
\text { amplitude V/I }\end{array}$ & $\begin{array}{l}\text { Alterado: Superior } \\
\text { a } 2 \text { desvios-padrão } \\
\text { da média dos } \\
\text { controles em cada } \\
\text { latência absoluta }\end{array}$ & $\begin{array}{l}\text { Teste de Fisher- } \\
\text { Behrens(estatística } \\
\text { d); frequência de } \\
\text { sujeitos classificados } \\
\text { com pelo menos uma } \\
\text { latência absoluta } \\
\text { alterada }\end{array}$ & $\begin{array}{l}\text { Em } 19 \text { de } 20 \text { sujeitos } \\
\text { com DP as latências } \\
\text { absolutas estão } \\
\text { normais }\end{array}$ & 7 & $\begin{array}{l}\text { Observou em um único caso o } \\
\text { aumento de latência e redução } \\
\text { da razão de amplitude } V / I \text {. }\end{array}$ \\
\hline $\begin{array}{l}\text { O'Donnel } \\
\text { et al., } \\
1987\end{array}$ & $\begin{array}{l}\text { 1) Intensidade em } 60 \mathrm{dBNS} \text {; } \\
\text { velocidade } 21 \text { clicks } / \mathrm{s} \text {; } \\
\text { 2) intensidade em } 75 \\
\mathrm{dBNS} \text {; velocidade } 21 \text { clicks } / \mathrm{s} \text {; } \\
\text { 3) intensidade em } 75 \mathrm{dBNS} ; \\
\text { velocidade } 51 \text { clicks } / \mathrm{s} \text {; } \\
\text { 4) intensidade em } 75 \mathrm{dBNS} \text {; } \\
\text { velocidade } 81 \text { clicks } / \mathrm{s}\end{array}$ & $\begin{array}{l}\text { Latências } \\
\text { absolutas das } \\
\text { ondas I e V }\end{array}$ & Não considerou & ANOVA & $\begin{array}{l}\text { Latência absoluta da } \\
\text { onda I semelhante } \\
\text { entre os grupos. }\end{array}$ & 3 & $\begin{array}{l}\text { Não analisa a latência interpico } \\
\text { que é o parâmetro mais } \\
\text { consistente. Não apresenta os } \\
\text { valores obtidos na latência da } \\
\text { onda I }\end{array}$ \\
\hline $\begin{array}{l}\text { Pineroli et } \\
\text { al., } 2002\end{array}$ & $\begin{array}{l}\text { Estímulo ipsilateral em } 40 \text { a } 50 \text { NS } \\
\text { aumentando a intensidade se não } \\
\text { tivesse ondas identificáveis- } \\
\text { velocidade em } 20 \text { cliks/s }\end{array}$ & $\begin{array}{l}\text { Latências } \\
\text { absolutas I, III } \\
\text { e V; latências } \\
\text { interpicos I-III, } \\
\text { III-V e I-V; }\end{array}$ & $\begin{array}{l}\text { Latência absoluta } \\
\text { da onda I 1,5 a 1,8; } \\
\text { onda III } 3,5 \text { a } 3,8 ; \\
\text { onda V 5,5 a } 6 \text { ms; } \\
\text { interpicos } 2 \text { a } 2,2 \\
\text { ms }\end{array}$ & $\begin{array}{l}\text { Frequência de } \\
\text { sujeitos classificados } \\
\text { como alterado }\end{array}$ & $\begin{array}{l}\text { Observou medidas } \\
\text { consideradas dentro } \\
\text { da normalidade nas } \\
\text { latências absolutas e } \\
\text { interpicos em todos os } \\
\text { sujeitos analisados }\end{array}$ & 8 & $\begin{array}{l}\text { Excluiu nove pacientes por } \\
\text { interferência nos traçados }\end{array}$ \\
\hline $\begin{array}{l}\text { Vitale et } \\
\text { al., } 2012\end{array}$ & $\begin{array}{l}\text { Estimulo ipsilateral em } 80 \mathrm{dBNS} \text {; } \\
\text { velocidade } 10 \text { clicks/s; polaridade } \\
\text { alternada; mascaramento em - } \\
40 \mathrm{~dB} \text {. }\end{array}$ & $\begin{array}{l}\text { Latências } \\
\text { absolutas I, III } \\
\text { e V; latências } \\
\text { interpicos I-III, } \\
\text { III-V e I-V }\end{array}$ & $\begin{array}{l}\text { Não apresenta a } \\
\text { informação }\end{array}$ & $\begin{array}{l}\text { Não apresenta a } \\
\text { informação }\end{array}$ & $\begin{array}{l}\text { Classifica como } \\
\text { normais os resultados } \\
\text { do PEATE de todos os } \\
\text { sujeitos com DP }\end{array}$ & 7 & $\begin{array}{l}\text { Não apresenta adequadamente } \\
\text { os resultados }\end{array}$ \\
\hline
\end{tabular}

Fonte: Elaboração da autora Legenda: ${ }^{* *}$ Critérios de Downs e Black ${ }^{9}$

Tabela 3. Estudos que identificaram respostas alteradas no Potencial Evocado Auditivo do Tronco Encefálico em sujeitos com DP.

\begin{tabular}{|c|c|c|c|c|c|c|c|c|c|}
\hline \multirow[t]{2}{*}{ Referência } & \multirow{2}{*}{$\begin{array}{l}\text { Protocolo de } \\
\text { aquisiçãa PEATE }\end{array}$} & \multirow[t]{2}{*}{ Variáveis analisadas } & \multirow{2}{*}{$\begin{array}{c}\text { Critério de } \\
\text { classificação } \\
\text { Normal/Alterado }\end{array}$} & \multicolumn{4}{|c|}{ Resultados } & \multirow[t]{2}{*}{ Pontuação** } & \multirow[t]{2}{*}{ Comentários } \\
\hline & & & & & $\mathrm{DP}$ & & ntroles & & \\
\hline $\begin{array}{l}\text { Gawel et } \\
\text { al., } 1981\end{array}$ & $\begin{array}{l}\text { Estímulo ipsilateral } \\
\text { em } 70 \mathrm{dBNS} \\
\text { velocidade } 10 \\
\text { clicks/s }\end{array}$ & $\begin{array}{l}\text { Latência absoluta e } \\
\text { amplitude das ondas } \\
\text { I e V }\end{array}$ & Não considerou & $\begin{array}{l}\text { Latência } \\
\text { Direita } \\
\text { Esquerd }\end{array}$ & $\begin{array}{l}\text { absoluta V } \\
5,91( \pm 0,19)^{*} \\
\text { a } 5,91( \pm 0,27)^{*}\end{array}$ & $\begin{array}{l}\text { Latência } \\
\text { Direita } \\
\text { Esquerda }\end{array}$ & $\begin{array}{l}\text { absoluta } V \\
5,69( \pm 0,18) \\
5,73( \pm 0,27)\end{array}$ & 5 & $\begin{array}{l}\text { Não considera para a análise o valor do } \\
\text { interpico I-V. Relata que as ondas nos } \\
\text { sujeitos com DP tem morfologia pior que } \\
\text { nos controles }\end{array}$ \\
\hline $\begin{array}{l}\text { Podoshin } \\
\text { et al., } \\
1987\end{array}$ & $\begin{array}{l}\text { Estimulo } \\
\text { ipsilateral; } \\
\text { velocidade em } 10 \\
\text { e } 55 \text { clicks } / \mathrm{s}\end{array}$ & $\begin{array}{l}\text { Latências absolutas } \\
\text { das ondas I, III e V; } \\
\text { latências interpicos I- } \\
\text { III, II+V e I-V; } \\
\text { diferença entre duas } \\
\text { velocidades dos } \\
\text { estímulos }\end{array}$ & Não considerou & $\begin{array}{l}\text { Interpico } \\
\text { Sem Trat } \\
\text { I-III } \\
\text { IIIV } \\
\text { I-V } \\
\text { Com trat } \\
\text { IIIV } 1\end{array}$ & $\begin{array}{l}\text { ts } \\
\text { tamento } \\
2,30( \pm 0,25)^{*} \\
2,01( \pm 0,27)^{*} \\
4,30( \pm 0,33)^{*} \\
\text { tamento } \\
1,81( \pm 0,49)^{*}\end{array}$ & $\begin{array}{l}\text { Interpicos } \\
\text { I-III } \\
\text { IIHV } \\
\text { I-V }\end{array}$ & $\begin{array}{l}\text { s } \\
2,17( \pm 0,24) \\
1,92( \pm 0,14) \\
4,10( \pm 0,26)\end{array}$ & 5 & $\begin{array}{l}\text { Não descreve a intensidade do estímulo } \\
\text { utilizada na pesquisa do PEATE; } \\
\text { Detectou diferenças entre DP não } \\
\text { tratado e controles em todos os } \\
\text { interpicos e DP não tratados e tratados } \\
\text { no interpico III-V; } \\
\text { Não houve diferença em relação às duas } \\
\text { velocidades do estimulo }\end{array}$ \\
\hline $\begin{array}{l}\text { Tachibana, } \\
\text { Takeda e } \\
\text { Sugita, } \\
1988\end{array}$ & $\begin{array}{l}\text { Estímulo ipsilateral } \\
\text { em } 60 \mathrm{dBNS} \text {; } \\
\text { mascaramento em } \\
-20 \mathrm{~dB}\end{array}$ & $\begin{array}{l}\text { Latências absolutas } \\
\text { das ondas I, III e V; } \\
\text { interpicos I-III, IIHV e } \\
\text { I-V; diferencial } \\
\text { interaural }\end{array}$ & Não considerou & $\begin{array}{l}\text { Interpico } \\
\text { IIIV } \\
\text { I-V }\end{array}$ & $\begin{array}{l}1,97( \pm 0,15)^{*} \\
4,14( \pm 0,20)^{*}\end{array}$ & $\begin{array}{l}\text { Interpico } \\
\mathrm{IIHV} \\
\mathrm{I}-\mathrm{V}\end{array}$ & $\begin{array}{l}\text { s } \\
1,83( \pm 0,13) \\
3,99( \pm 0,18)\end{array}$ & 7 & \\
\hline $\begin{array}{l}\text { Fradis et } \\
\text { al., } 1988\end{array}$ & $\begin{array}{l}\text { Estímulo ipsilateral } \\
\text { em } 75 \text { dBNA; } \\
\text { velocidade em } 10 \\
\text { e } 55 \text { clicks/s; } \\
\text { polaridade } \\
\text { alternada; }\end{array}$ & $\begin{array}{l}\text { Latências interpicos } \\
\text { I-III, IIHV e I-V; } \\
\text { diferença entre as } \\
\text { duas velocidades de } \\
\text { apresentação dos } \\
\text { estímulos }\end{array}$ & Não considerou & $\begin{array}{l}\text { Interpico } \\
\text { I-III } \\
I I-V \\
I-V\end{array}$ & $\begin{array}{l}\text { 2,30( } \pm 0,26)^{*} \\
2,01( \pm 0,27)^{*} \\
4,30( \pm 0,33)^{*}\end{array}$ & $\begin{array}{l}\text { Interpicos } \\
\text { I-III } \\
\text { IIIV } \\
\text { I-V }\end{array}$ & $\begin{array}{l}\text { s, } \\
2,17( \pm 0,24) \\
1,92( \pm 0,14) \\
4,10( \pm 0,25)\end{array}$ & 7 & \\
\hline $\begin{array}{l}\text { Tachibana, } \\
\text { Takeda e } \\
\text { Sugita, } \\
1989\end{array}$ & $\begin{array}{l}\text { Estímulo ipsilateral } \\
\text { em } 60 \mathrm{dBNS} ; \\
\text { velocidade } 10 \\
\text { clicks/s; } \\
\text { mascaramento }-20\end{array}$ & $\begin{array}{l}\text { Latências absolutas } \\
\text { das ondas I, III, V; } \\
\text { latências interpicos I- } \\
\text { III, II+V e I-V }\end{array}$ & Não considerou & $\begin{array}{l}\text { Interpico } \\
\text { IIIV } \\
\text { I-V } \\
\text { *Demên } \\
\text { I-V }\end{array}$ & $\begin{array}{l}1,94( \pm 0,14)^{*} \\
4,13( \pm 0,20)^{*} \\
4,26( \pm 0,19)^{*}\end{array}$ & $\begin{array}{l}\text { Interpico } \\
\mathrm{IIHV} \\
\mathrm{I}-\mathrm{V}\end{array}$ & $\begin{array}{l}s \\
1,86( \pm 0,13) \\
4,02( \pm 0,17)\end{array}$ & 8 & \\
\hline $\begin{array}{l}\text { Chia et al., } \\
1995\end{array}$ & $\begin{array}{l}\text { Não descreve o } \\
\text { protocolo } \\
\text { aplicado. }\end{array}$ & $\begin{array}{l}\text { Latências absolutas } \\
\text { das ondas I, II, III, IV } \\
\text { e V; latência } \\
\text { interpico I-V }\end{array}$ & Não considerou & $\begin{array}{l}\text { Interpico } \\
\text { Com levc } \\
4,21( \pm 0, \\
\text { Sem levo } \\
4,30( \pm 0,\end{array}$ & $\begin{array}{l}\text { o-V } \\
\text { odopa } \\
29)^{*} \\
\text { odopa } \\
26)^{*}\end{array}$ & $\begin{array}{l}\text { Interpico } \\
4,06( \pm 0,1\end{array}$ & & 8 & $\begin{array}{l}\text { Faz referência a protocolo aplicado em } \\
\text { estudo anterior indisponível. }\end{array}$ \\
\hline $\begin{array}{l}\text { Yýlmaz, et } \\
\text { al., } 2009\end{array}$ & $\begin{array}{l}\text { Estímulo ipsilateral } \\
\text { em } 80 \mathrm{dBNA} ; \\
\text { velocidade } 10 \\
\text { clicks/s; } \\
\text { polaridade } \\
\text { alternada; } \\
\text { mascaramento em } \\
\text {-40dB. }\end{array}$ & $\begin{array}{l}\text { Latências absolutas } \\
\text { das ondas I, III e V; } \\
\text { latências interpicos I- } \\
\text { III, II+V e I-V }\end{array}$ & Não considerou & $\begin{array}{l}\text { Latência } \\
\text { Onda V } \\
\text { Interpico } \\
\text { I-V }\end{array}$ & $\begin{array}{l}\text { absoluta } \\
6,04( \pm 0,41) \\
4,45( \pm 0,46)\end{array}$ & $\begin{array}{l}\text { Latência } \\
\text { Onda V } \\
\text { Interpico } \\
\text { I-V }\end{array}$ & $\begin{array}{l}\text { absoluta V } \\
5,87( \pm 0,31) \\
4,23( \pm 0,38)\end{array}$ & 6 & \\
\hline
\end{tabular}

Fonte: Elaboração da autora Legenda: * Teste T de Student ${ }^{* *}$ Critérios de Downs e Black ${ }^{9}$ 
te significantes (Tabela 3). Cabe salientar que Tachibana, Takeda e Sugita ${ }^{17}$ verificaram que este prolongamento ocorreu somente nos sujeitos com diagnóstico de demência.

A análise da qualidade metodológica dos estudos evidenciou pontuação média de 6,4 , sendo o valor mínimo três e máximo de oito pontos. Salienta-se que nenhum estudo atingiu 50\% da pontuação máxima, sendo o melhor resultado encontrado nos estudos de Tachibana, Takeda e Sugita ${ }^{17}$, Chia et al.18 e Pinerolli et al. ${ }^{19}$, seguido por quatro estudos que atingiram sete pontos ${ }^{12,15,16,21}$. Destes sete estudos que apresentaram maior pontuação, quatro apontam para a presença de alterações no PEATE em indivíduos com DP.

$\mathrm{Na}$ análise do critério quanto à seleção dos sujeitos para participação no estudo, verificou-se que todas as investigações foram conduzidas com amostras por conveniência, isto é, nenhum processo probabilístico foi aplicado para a seleção dos sujeitos. Adicionalmente, em nenhum estudo houve a indicação de que os controles foram selecionados a partir da mesma população dos sujeitos com DP, e somente Vitale et al. ${ }^{21}$ identificaram o mesmo período para a identificação dos indivíduos com e sem DP incluídos no estudo. Desta forma, considerou-se que em todos os estudos foram analisadas amostras não representativas da população de origem na constituição dos grupos DP e controle.

Quanto à aferição da exposição, a descrição do critério diagnóstico e caracterização do quadro clínico da DP não foram realizadas em dois estudos ${ }^{11,14}$ ou foi parcialmente abordada em quatro deles ${ }^{12,13,14,20}$. Em relação ao item que avalia a descrição da distribuição de confundidores entre os grupos avaliados, observou-se que apenas um estudo considerou na análise dos resultados a variável quadro cognitivo ${ }^{17}$ e em outros dois foi considerada a terapia dopaminérgica ${ }^{14,18}$.

\section{DISCUSSÃO}

Nesta revisão, identificamos controvérsias quanto a presença de processos fisiopatológicos na atividade neural das vias auditivas aferentes na DP, avaliadas pela pesquisa do PEATE. Existem poucas investigações sobre o tema e acredita-se que as diferentes análises dos desfechos, bem como as importantes limitações metodológicas verificadas, possam responder pelas divergências nas conclusões dos autores.

O prolongamento das latências do PEATE foi identificado em alguns estudos ${ }^{12,14,15,16,17,18,20}$, e sugere a lentificação da condução neuroauditiva na porção correspondente ao tronco encefálico nos sujeitos com DP. Contudo, o método utilizado para estimativa do desfecho principal impõe análises cautelosas quanto à conclusão apresentada.

Gawel et al. ${ }^{11}$ compararam a latência absoluta da onda $\mathrm{V}$, evocada por estímulos $70 \mathrm{~dB}$ nível de sensação, mas não relataram a investigação do quadro audiológico dos sujeitos avaliados, o que pode ter colaborado para os resultados obtidos. Sabe-se que as latências, em valores absolutos, são influenciadas pela intensidade de apresentação do estímulo em confluência com aspectos audiológicos, como alterações de orelha média e perdas auditivas sensoriais. Em alterações que comprometem a transdução do som realizada pela orelha média, as latências absolutas das ondas I, III e V podem estar significativamente aumentadas ${ }^{22}$. De forma não tão proeminente, o prolongamento das latências também pode ocorrer em perdas auditivas sensoriais com piores limiares em frequências agudas a partir de $1000 \mathrm{~Hz}$ ou 2000 $\mathrm{Hz}^{22}$.

Nos demais estudos, foi reportada diferença estatisticamente significante, calculada através do teste $T$ Student, entre as médias e desvios-padrão dos interpicos I-III, III-V e I-V ${ }^{14,16}$, dos interpicos III-V e I-V ${ }^{15,17}$ ou somente do interpico I-V ${ }^{18,20}$. Entretanto, não foi identificada nos estudos a informação de processo de amostragem aleatória ou distribuição normal dos valores das variáveis analisadas, requisitos para aplicação do teste T Student. Além disso, observam-se diferenças sutis das médias dos interpicos entre os grupos e, caso seja considerado o desvio-padrão desta medida, verifica-se valores comuns ao grupo DP e ao grupo controle. Adicionalmente, é importante salientar que esse tipo de análise do desfecho principal não permite caracterizar quais os padrões de resposta esperados clinicamente para sujeitos com DP e, tampouco, permitem inferências sobre a frequência de sujeitos com comprometimento da condução neuroeletrofisiológica auditiva.

Contrapondo estes achados, outros estudos observaram respostas do PEATE normais nos sujeitos com DP 12,13,19,21. Em três destas investigações foi aplicado um critério classificatório das respostas de cada indivíduo analisado, considerando um padrão de normalidade estabelecido por dados da literatura ${ }^{19}$ ou de acordo com as distribuições dos valores das respostas obtidas em um grupo controle ${ }^{12,21}$.

Prasher e Bannister ${ }^{12}$ analisaram as latências absolutas das ondas I, III e V, assim como a razão de amplitude entre as ondas V e I. Conforme exposto anteriormente, o prolongamento nas latências absolutas pode ocorrer devido a fatores adjacentes aos processos fisiopatológicos da doença, como o protocolo de intensidade do estímulo e características audiológicas dos sujeitos. Da mesma forma que no estudo de Gawel et al. ${ }^{11}$, especula-se que estes fatores podem ter influenciado os resultados do estudo de Prasher e Bannister ${ }^{12}$, porém em direção contrária a identificação de possíveis alterações.

Na prática clínica, recomenda-se que a identificação do comprometimento da condução neuroeletrofisiológica das vias auditivas seja realizada considerando, principalmente, a análise das latências interpicos das ondas I-III, III-V e I-V ${ }^{23}$ por serem os parâmetros menos influenciados por fatores adjacentes como a idade, velocidade de apresentação dos estímulos e redução dos níveis auditivos periféricos ${ }^{24}$. Com base nestes aspectos, os estudos de Pinerolli et al. ${ }^{19} \mathrm{e}$ Vitale et al. ${ }^{21}$ empregaram medidas mais adequadas para a análise do desfecho principal. 
Por outro lado, O'Donnel et al. ${ }^{13}$, analisando a latência absoluta da onda I, também verificaram PEATE normal em sujeitos com DP, sugerindo que a resposta neural da porção distal do nervo vestíbulo-coclear não está comprometida na DP. Este achado deve ser interpretado com cautela, pois alterações do nervo vestíbulo- coclear não são esperadas na DP, já que a base das alterações sensoriais da doença ocorrem no tronco encefálico em direção às vias corticais.

Cada uma das ondas identificadas no PEATE reflete o volume de resposta bioelétrica gerado por mudanças no potencial de membrana de uma população neuronal, organizada em regiões específicas da via auditiva. A latência absoluta, caracterizada em milissegundos, traduz o tempo decorrido entre a apresentação do estímulo e a geração do pico de energia do agrupamento neuronal em um ponto determinado. Já a latência interpico é um valor relativo, e remete ao tempo decorrido na geração da resposta bioelétrica entre duas estações neuronais da via auditiva ascendente ${ }^{23}$. Desta forma, o aumento da latência interpico sugere o comprometimento dos processos de sincronia na despolarização dos axônios destas células e da transdução sináptica entre grupos neuronais, localizados nas diferentes estações da via auditiva ascendente ${ }^{9}$. Mecanismos neuropatológicos que envolvam processos desmielinizantes e redução de neurotransmissores, podem comprometer a condução neurofisiológica desta via ${ }^{25,26,27 .}$

A redução generalizada de neurotransmissores foi apontada como uma possível condição fisiopatológica para alterações do PEATE em sujeitos com DP11,15,18. Sabe-se que os neurotransmissores colinérgicos, serotoninérgicos e noradrenérgicos estão implicados na modulação das respostas neuronais das vias auditivas do tronco encefálico, especialmente na região do núcleo coclear e colículo inferior ${ }^{27,28}$. Desta forma, é possível hipotetizar que a redução destes neurotransmissores compromete a atividade sináptica dos neurônios, aumentando o tempo de resposta das vias auditivas no tronco encefálico alto.

Adicionalmente, Tachibana, Takeda e Sugita ${ }^{17}$ acrescentam a esta hipótese o papel da demência na DP, como parte de um quadro que traduz comprometimentos mais generalizados na doença. A fisiopatologia das alterações cognitivas na DP ainda não é claramente estabelecida. Contudo, estudos apontam para uma associação com a presença de inclusões citoplasmáticas de agregados da proteína alfa-sinucleína (corpúsculos de Lewy) no núcleo basal de Meynert e Locus Coeruleus ${ }^{29}$, que são estruturas conectadas ao tronco encefálico pela projeção de fibras colinérgicas e noradrenérgicas. ${ }^{27,28}$ Desta forma, acredita-se que o quadro cognitivo dos sujeitos avaliados pode ter contribuído para achados controversos entre os estudos, uma vez que, em oposição aos resultados de Tachibana, Takeda e Sugita ${ }^{17}$, os estudos que excluíram sujeitos com demência ${ }^{19,21}$ apontam para respostas do PEATE normais na DP.
Por outro lado, Podoshin et al. ${ }^{14}$ e Fradis et al. ${ }^{16}$ sugerem que o sistema dopaminérgico não apresenta influência na velocidade da transmissão sináptica destas vias, observando resultados semelhantes nas pesquisas realizadas com estímulo em 10 e 55 clicks/segundo, em sujeitos com DP não tratados e tratados com reposição dopaminérgica. Contudo, propõem que as diferentes respostas do PEATE observadas em seu estudo teriam relação com os efeitos vasomotores desta terapia. Segundo os autores, os baixos níveis de levodopa promoveriam a vasoconstricção na região do tronco encefálico alto, o que levaria a uma alteração temporária da condução nervosa da substância branca nos indivíduos sem tratamento. Da mesma forma, reforçam a hipótese do efeito vasomotor da levodopa, atribuindo à sua ação vasodilatadora o encurtamento das respostas verificado nos sujeitos tratados. O'Donnel et al. ${ }^{13}$ também referem um possível efeito do tratamento dopaminérgico para a explicação dos resultados normais no PEATE observados nos sujeitos com DP sob tratamento, entretanto, não abordam de que forma essa influência ocorreria. Contudo, os achados de Chia et al. ${ }^{18}$ não corroboram essa hipótese, pois observaram prolongamento das latências em sujeitos tratados e não tratados.

Cabe salientar ainda que a terapia de reposição dopaminérgica é o tratamento padrão-ouro para a DP, com eficácia bem estabelecida na redução dos sinais motores na doença ${ }^{30}$. No entanto, seus efeitos no sistema auditivo ainda são controversos ${ }^{31,32}$, havendo a necessidade de condução de novos estudos que abordem essa temática.

Os resultados da avaliação da qualidade metodológica dos estudos reforçam a fragilidade dos achados obtidos nas investigações por possível influência de vieses de seleção, aferição e confundimento. Acredita-se que as amostras por conveniência são hipoteticamente mais suscetíveis ao viés de seleção, uma vez que a representatividade da população de origem pode ser limitada ou tendenciosa quanto à distribuição das variáveis investigadas. A ausência de um processo aleatório na seleção dos sujeitos que participaram do estudo pode favorecer, por exemplo, a inclusão de um maior número de indivíduos com alguma queixa relacionada ao desfecho investigado ou daqueles com quadros clínicos mais graves, acompanhados com maior frequência nos centros especializados.

A adequada aferição da exposição e definição de desfechos de análise reflete diretamente na qualidade metodológica do estudo e visam dirimir ao máximo a possibilidade de vieses. Neste aspecto, acredita-se que a descrição, inexistente ou imprecisa, de como a exposição ao fator DP idiopática foi estabelecida em alguns estudos leva à suspeição da presença do viés de aferição. Ademais, essa fragilidade metodológica, somada aos diferentes métodos de análise do desfecho principal, compromete a realização de uma metanálise dos estudos. Cabe salientar ainda, que mesmo considerando as inves- 
tigações conduzidas com maior rigor metodológico $^{12,15,16,17,18,19,21}$, os achados apresentados são controversos e impõem limite a conclusões definitivas sobre o tema.

Outro aspecto de extrema relevância diz respeito ao viés de confundimento. Para a análise dos estudos quanto ao estabelecimento de variáveis confundidoras, foram elencadas as variáveis diabetes, doença cardiovascular e hiperlipidemia, por sua elevada prevalência em sujeitos idosos em geral, sem relação direta com o processo fisiopatológico da $\mathrm{DP}$, e que podem comprometer a condução nervosa das vias auditivas investigadas no PEATE ${ }^{33,34}$. A demência, também frequente em idosos, se configura com um possível modificador de efeito da exposição, pois tem relação com comprometimentos difusos dos processos fisiopatológicos da doença $^{29}$. Com relação à terapia de reposição dopaminérgica, sabe-se que está diretamente implicada na DP por sua eficácia na melhora do quadro motor ${ }^{30}$ e, embora seu efeito no sistema auditivo ainda não tenha sido claramente estabelecido, considerá-la como um possível modificador de efeito das respostas eletrofisiológicas é uma medida metodologicamente pertinente.

Apenas três estudos analisados controlaram seus resultados pela variável quadro cognitivo ${ }^{17}$ ou terapia dopaminérgica ${ }^{14,18}$. As variáveis diabetes ${ }^{13,14,16,20,21}$, hiperlipidemia ${ }^{14,16,21}$, doença cardiovascular ${ }^{11,13,21}$ e demência ${ }^{19,21}$ foram consideradas como fatores de exclusão e, consequentemente, suas distribuições entre os grupos não foram investigadas. Adicionalmente, Podoshin et al. ${ }^{14}$ não consideraram essas variáveis sob nenhum dos aspectos anteriormente descritos.

Desta forma, é possível supor que o tratamento dos dados em relação aos possíveis confundidores ou modificadores de efeito, aplicado de forma parcial naqueles estudos que controlaram os resultados por apenas uma das variáveis propostas, ou que inadequadamente as utilizaram como fatores de exclusão, possa ter contribuído significativamente para as conclusões controversas observadas.

Contudo, é importante salientar que a condução de pesquisas nessa temática não se constitui uma tarefa simples. As investigações audiológicas requerem o emprego de condições de teste específicas, com equipamentos de alta tecnologia e ambientes especialmente preparados, o que dificulta sua realização em ambulatórios clínicos de neurologia, havendo a necessidade de deslocamento dos sujeitos para os serviços de audiologia. Considerando os comprometimentos motores decorrentes da DP, os quais prejudicam em diferentes graus a independência dos doentes, em associação com outras limitações decorrentes do envelhecimento, é possível imaginar a complexidade logística na condução destes estudos, o que pode responder pelo número limitado de indivíduos participantes. Além disso, a contaminação de artefatos musculares nos sinais aquisitados, bem como a imprecisão e comprometimento da morfo- logia das ondas, são aspectos recorrentes que tornam o procedimento de coleta uma atividade complexa que requer tempo e habilidade por parte dos examinadores. Desta forma, entende-se que as investigações abordadas nesta revisão são relevantes para o entendimento do tema em questão e para o provimento de informações que nortearão pesquisas futuras.

Sendo assim, acredita-se que resultados conclusivos possam ser estabelecidos com a aplicação de parâmetros clínicos para a identificação dos resultados do PEATE, assim como uma melhor descrição e tratamento das variáveis de confundimento e de modificação de efeito. Da mesma forma, sugere-se a implementação de cuidados metodológicos no que se refere à seleção de sujeitos oriundos de uma mesma população para a constituição dos grupos de análise.

\section{CONCLUSÃO}

A presença de processos que comprometam a condução neuroeletrofisiológica das vias auditivas aferentes é controversa em sujeitos com doença de Parkinson, não sendo possível estabelecer, até o presente momento, um padrão que configure claramente alterações do Potencial Evocado Auditivo do Tronco Encefálico. Desta forma, futuras investigações clínicas e experimentais são necessárias para esclarecer a epidemiologia do comprometimento neuroauditivo na região do tronco encefálico em sujeitos com DP, bem como o processo fisiopatológico envolvido nestas alterações.

\section{REFERÊNCIAS}

1. IBGE. Instituto Brasileiro de Geografia e Estatística. Censo 2010. Disponível em: <http://censo2010.ibge.gov.br/>. Acesso em: 14 set. 2013.

2. DE RIJK, M. C. et al. Prevalence of Parkinson's disease in Europe: a collaborative study of population-based cohorts: neurologic diseases in the elderly research group. Neurology, Minneapolis, v. 54, n. 11, p. 21-23, 2000.

3. LIMONGI, J. C. P. Principais sintomas, causas e formas clínicas. In: LIMONGI, J. C. P. (Org.). Conhecendo melhor a doença de Parkinson: uma abordagem multidisciplinar com orientações práticas para o dia-a-dia. São Paulo: Plexus, 2001. p. 13-35.

4. CHAUDHURI, K. R.; HEALY, D. G.; SCHAPIRA, A. H. Non-motor symptons of Parkinson's disease: diagnosis and management. Lancet Neurol., London, v. 5, n. 3, p. 235-245, Mar. 2006.

5. MATTOS, L. C.; VERAS, R. P. Prevalência da perda auditiva em uma população de idosos da cidade do Rio de Janeiro: um estudo seccional. Rev. Bras. Otorrinolaringol., São Paulo, v. 73, n. 5, p. 654-659, set./out. 2007

6. ROTH, N. T.; HANEBUTH, D.; PROBST, R. Prevalence of age-related hearing loss in Europe: a review. Eur. Arch. Otorhinolaryngol., Bonn, v. 268, n. 8, p. 1101-1107, Aug. 2011.

7. SOMMERS, M. S., et al. Listening comprehension across the adult lifespan. Ear Hear., Pennsville, v. 32, n. 6, p. 775-781, Nov./Dec. 2011.

8. LI-KOROTKY, H. S. Age-related hearing loss: quality of care for quality of life. The Geront., Washington, v. 52, n. 2, p. 265-271, 2012. 
9. BURKARD, R. F.; DON, M. The auditory brainstem response. In: BURKARD, R. F.; DON, M.; EGGERMONT, J. J. (Org). Auditory evoked potentials: basic principles and clinical application. Philadelphia: Lippincott Williams \& Wilkins, 2007. p. 229-250.

10. DOWNS, S. H.; BLACK, N. The feasibility of creating a checklist for the assessment of the methodological quality both of randomized and non-randomized studies of health care interventions. J. Epidemiol. Community Health, London, v. 52, n. 6, p. 377-384, 1998

11. GAWEL, M. J. et al. Visual and auditory evoked responses in patients with Parkinson's disease. J. Neurol. Neurosurg. Psychiatry., London, v. 44, n. 3, p. 227-232, Mar. 1981

12. PRASHER, D.; BANNISTER, R. Brain stem auditory evoked potentials in patients with multiple system atrophy with progressive autonomic failure (Shy-Drager syndrome). J. Neurol. Neurosurg. Psychiatry., London, v. 49, n. 3, p. 278-289, Mar. 1986.

13. O'DONNEL, B. F. et al. Evoked potential changes and neuropsychological performance in Parkinson's disease. Biol. Psychol., Amsterdan, v. 24, n. 23-37, Feb. 1987.

14. PODOSHIN, L. et al. Brainstem auditory evoked potentials with and without increased stimulus rate as diagnostic tool in brainstem minor transient changes. ORL J. Otorhinolaryngol Relat. Spec., Basel, v. 49, n. 6, p. 287-293, 1987.

15. TACHIBANA, H.; TAKEDA, M.; SUGITA, M. Electrophysiological differences between parkinson's disease and vascular parkinsonism. Jpn. J. Med., Tokio, v. 27, n. 3, p. 261-266, Aug. 1988

16. FRADIS, M. et al. Brainstem auditory evoked potentials to different stimulus rates in parkinsonian patients. Eur. Neurol., Basel, v. 28, n. 4, p. $181-186,1988$

17. TACHIBANA, H.; TAKEDA, M.; SUGITA, M. Short-latency somatosensory and brainstem auditory evoked potentials in patients with Parkinson's disease. Int. J. Neurosci., London, v. 44, n. 3-4, p. 321-326, Feb. 1989.

18. CHIA, L. G., et al. Studies of dementia, depression, electrophysiology and cerebrospinal fluid monoamine metaboities in patients with Parkinson's disease. J. Neurol. Sci., Amsterdan, v. 133, n. 1-2, p. 73-78, Nov. 1995.

19. PINEROLI, J. C. A. et al. Avaliação auditiva central com BERA e P300 na doença de Parkinson. Rev. Bras. Otorrinolaringol., São Paulo, v. 68, n. 3, p. 462-466, jul./ago. 2002.

20. YÝLMAZ, S. et al. Auditory evaluation in Parkinsonian patients. Eur. Arch. Otorhinolaryngol., Bonn, v. 266, n. 5, p. 669-671, May. 2009.
21. VITALE, C. et al. Hearing impairment in Parkinson's disease: expanding the nonmotor phenotype. Mov. Disord., New York, v. 12, n. 12, p. 1530-1535, Oct. 2012.

22. STEINHOFF, H. J.; BÖHNKE, F.; JANSSEN, T. Click ABR intensity-latency characteristics in diagnosing conductive and cochlear hearing losses. Arch. Otorhinolaryngol., Bonn, v. 245, n. 5, p. 259-265, 1988.

23. AMERICAN CLINICAL NEUROPHYSIOLOGY SOCIETY. Guideline 9C: guidelines on short-latency auditory evoked potentials. J. Clin. Neurophysiol., New York, v. 23, n. 2, p. 157-167, Apr. 2006

24. KONRAD-MARTIN, D. et al. Age-related changes in the auditory brainstem response. J. Am. Acad. Audiol., Hamilton, v. 23, n. 1, p. 1835, Jan. 2012.

25. SABERI, A. et al. Hearing statement in multiple sclerosis: a case control study using auditory brainstem responses. Acta Med. Iran., Tehran, v. 50, n. 10, p. 679-683, 2012.

26. GAZULA, V. R. et al. Localization of Kv1.3 channels in presynaptic terminals of brainstem auditory neurons. J. Comp. Neurol., New York, v. 518 , n. 16, p. $3205-3220,2010$

27. ASHKENAZI, A.; FREEMAN, S.; ARGOV, Z. Effects of cholinergic blockers on auditory brain-stem evoked potentials in rats. J. Neurol. Sci., Amsterdan, v. 164, n. 2, p. 124-128, Apr. 1999.

28. KLEPPER, A.; HERBERT, H. Distribution and origin of noradrenergic and serotonergic fibers in the cochlear nucleus and inferior colliculus of the rat. Brain Res., Amsterdan, v. 557, n. 1-2, p. 190-201, Aug. 1991.

29. EMRE, M. What causes mental dysfunction in Parkinson's disease? Mov. Disord., New York, v. 18, p. 63-71, Sep. 2003. Suppl. 6.

30. SCHAPIRA, A. H. Treatment options in the modern management of Parkinson disease. Arch. Neurol., Chicago, v. 64, n. 8, p. 1083-1088, Aug. 2007.

31. PRASHER, D.; FINDLEY, L. Dopaminergic induced changes in cognitive and motor processing in Parkinson's disease: an electophysiological investigation. J. neurol., Neurosurg. Psychiatry., London, v. 54, p. 603609, 1991.

32. PRABHAKAR, S.; SYAL, P.; SRIVASTAVA, T. P300 in newly diagnosed non-dementing Parkinson's disease: effect of dopaminergic drugs. Neurol. India, Hyderabad, v. 48, n. 3, p. 239-242, Sep. 2000.

33. GUPTA, S. et al. Brainstem auditory evoked potential abnormalities in type 2 diabetes mellitus. N. Am. J. Med. Sci.,Hamilton, v. 5, n. 1, p. 60-65, Jan. 2013

34. THAKUR, J. S. et al. Auditory brainstem evoked responses in hyperlipidaemia: effect of various lipid fractions on auditory function. J. Laryngol. Otol., London, v. 126, n. 3, p. 249-256, Mar. 2012

Submetido em 13.11.2013

Aceito em 20.12.2013. 